\title{
Uyghur -ip as a verb linker in multiple constructions
}

\author{
Alexander Sugar*
}

\begin{abstract}
Uyghur grammars and linguistic works have traditionally described the -ip morpheme as a suffix that derives adverbs from verbs (Tömür 2003, Tohti 2012).

This paper uses structural diagnostics involving passive morphology, single negation and NPI licensing to argue that -ip is a functional head with multiple syntactically distinct roles in different structures. The distinct properties of these structures, according to the types of verbs that are linked by -ip, provide evidence against a uniform derivational process of adverb formation. In some case -ip allows for adjunction of a TP-sized constituent, suggesting it may be a non-finite T head. In other cases it allows for adjunction of a verb phrase, and in yet another case it creates a lexical verb + auxiliary sequence in a monoclausal structure. I suggest that -ip in the latter two cases may be an event head.
\end{abstract}

Keywords. Uyghur; syntax; multi-verb constructions; serial verb constructions; auxiliaries; event; -ip; monoclausal

1. Introduction. The Turkic language Uyghur has a productive strategy of linking two verbal constituents within a sentence using the suffix -ip. This suffix appears in complementary distribution with tense inflection, and it is only the final verb in an -ip construction that must be inflected for tense and person. (1) shows the verb oyna 'to play' marked by -ip, followed by the inflected final verb qayt 'to return.'

(1) Uyghur (A. Turdimemet, p.c., 12/9/2016)

Ular meydan-da putbol oyna-ip yataq-ga qayt-di-0

3PL field-LOC soccer play-IP dorm-DAT return-PST-3

"They played soccer on the field, and came back to the dorm."

Previous analyses have treated -ip as the syntactic head of an adverbial phrase that selects a verbal complement. In this paper, I argue that a uniform analysis of -ip as a syntactic head is not possible, because -ip constructions have different structure depending on the relationship between the two verbs. Instead, -ip may be either a T or event head. Here I consider three different constructions in which a distinct relationship is established between a final and non-final verb through use of the -ip suffix (for alternative divisions of types, see Bridges 2008 or Tohti 2012). ${ }^{2}$

In the first type of construction, -ip can link two lexical verbs such that each verb denotes a separate event. In (1), for example, the actions of playing soccer on the field and returning to the dorm are understood to happen at two different times and places, and it is possible that the sentence glosses over some other minor event that occurred between the two, such as the actors

${ }^{*}$ Thank you to Edith Aldridge and Karen Zagona for feedback on earlier drafts of this paper. Also, thank you to the attendees of the 91st Meeting of the Linguistic Society of America for helpful comments on the poster. Finally, I am grateful to the judgments provided by Uyghur native speakers Abdurehim Turdimemet, Litip Tohti, Ahmatjan Tash, Mahire Yakup and Chughluk Abdilim, without whom this paper would not be possible. All errors are mine. Alexander Sugar, University of Washington (sugara@u.washington.edu).

${ }^{2}$ I refer to the non-final and final verbs respectively as V1 and V2 by virtue of their linear order. 
stopping to buy snacks on the way back to the dorm. I call this construction a multiple event construction.

-Ip can also link two lexical verbs whose meaning comprises a single event. In (2), qayt 'to return' and kel 'to come' collectively describe a single event that can be translated into English as "coming back," with kel indicating the direction of return. I call this construction a VV single event construction.

(2) Tursun qayt-ip kel-di-0

NAME return-IP come-PST-3

"Tursun came back." (A. Turdimemet, p.c., 12/9/2016)

The third type of -ip construction I will discuss consists of a lexical V1 followed by a V2 that has been semantically bleached of its lexical meaning (Ibrahim 1995). Instead of supplying a predication, V2 modifies the aspectual properties of the event denoted by V1, and in some cases adds information about the speaker or subject's attitude towards the event. The V2 tur in (3) does not mean 'to stand', but instead means that the action of writing denoted by V1 yaz keeps happening. I call this an auxiliary construction.
Tursun öy-i-ga
pat-pat xet yaz-ip tur-y-du
NAME home-3SG.POSS-DAT often letter write-IP stand-NPST-3
“Tursun often writes letters home.” (Tohti 2012: 360)

After discussing previous syntactic analyses of these three constructions, I will provide new tests showing that they differ in their clausal structure, leading to proposals of -ip as a different type of inflectional head and/or in a different structural relationship to the finite verb phrase in each construction.

2. Previous analyses. Previous discussions of Uyghur syntax have treated -ip as a head that selects verbal material and projects an adverbial phrase, regardless of the construction type in which it appears (Tömür 2003, Tohti 2012, Abdurusul 2014).

The most up-to-date discussions have been given by Tohti (2012) and Abudurusul (2014), who basically share the same analyses. Both authors treat -ip as an adverbializing head, and argue that the phrase it projects adjoins to V2P in multiple event constructions. For example, Abdurusul gives a sentence like (4) the analysis shown in (5).
Tursun dada-i-ni
kör-ip nahayiti xush bol-di-0
NAME father-3.POSS-ACC see-IP extremely happy become-PST-3

"Tursun saw his father and became extremely happy." (Abdurusul 2014: 236) 
(5)

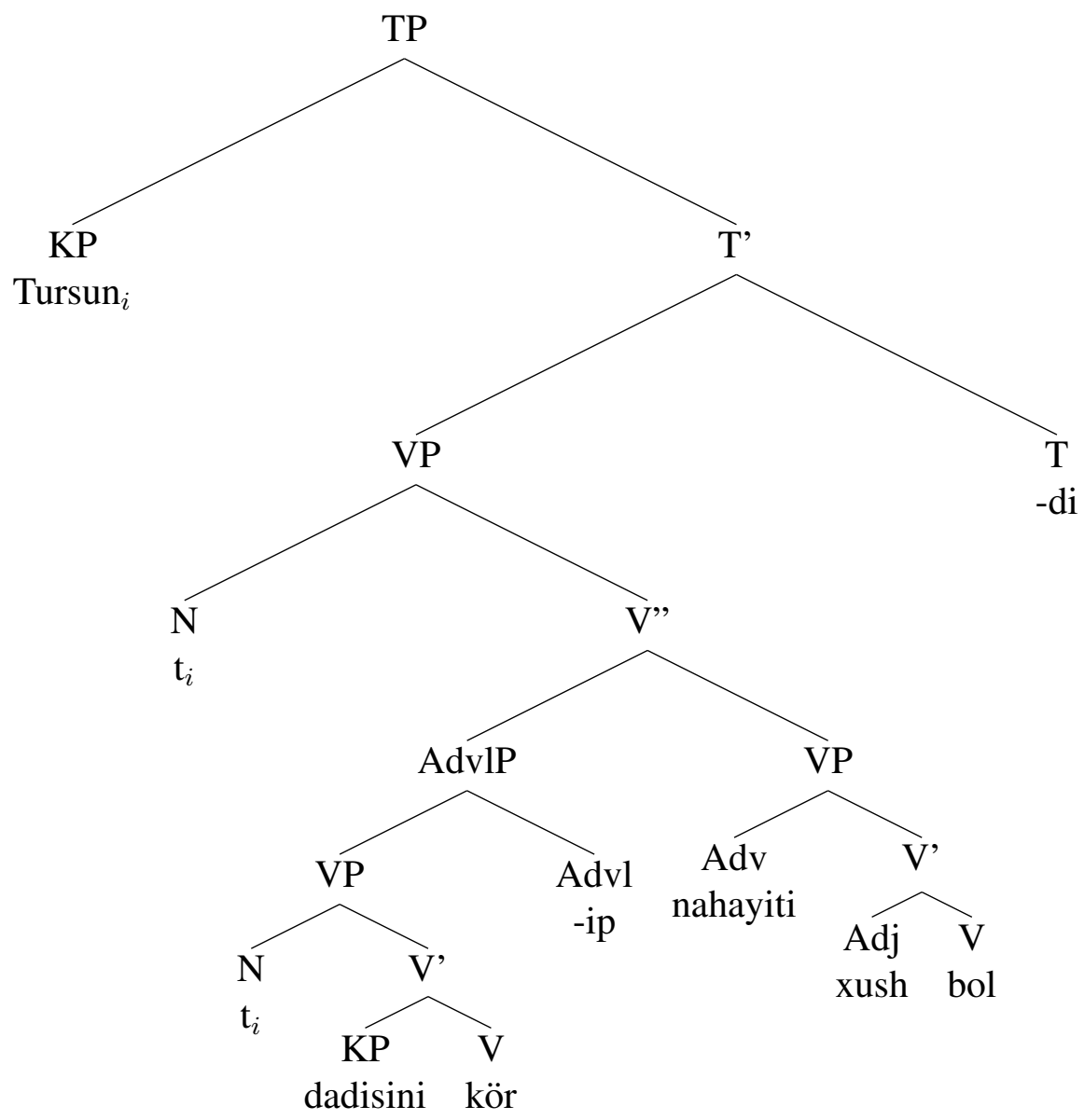

In (5), we see -ip heading an AdvlP that selects a VP, dadisini kör 'see his father', as its complement and adjoins to the VP nahayiti xush bol 'become extremely happy'. The subject Tursun in this structure was generated as the subject of V1, and moves through V2 on the way to its surface position, but Abdurusul also entertains the possibility that the subject controls a PRO in spec, VP.

The authors also label -ip as an adverbializing head in auxiliary constructions, but make clear from their tree representations that the phrase headed by -ip is the complement of the auxiliary (which they label an aspectual head), rather than an adjunct. Abdurusul analyzes a sentence like (6) as having the structure shown in (7).

(6) Tursun xet yaz-ip qoy-di-0

NAME letter write-IP put-PST-3

"Tursun wrote up a letter." (Abdurusul 2014: 233) 


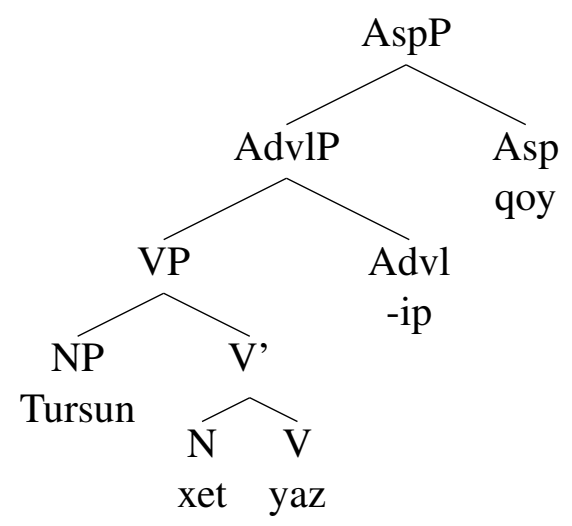

Here it is important to note that qoy and all other auxiliaries sit in an Aux head whose complement is the same -ip-headed adverbial phrase seen in (5). The structure leads one to assume that there is a single syntactic position on the clausal spine for all auxiliaries.

As for VV single event constructions, Tohti suggests that certain idiosyncratic verb combinations are formed in the lexicon, while Abdurusul suggests that they have the same structure as auxiliary constructions, with V2 in a VV single event construction selecting an -ip-headed adverbial phrase as its complement. A syntactic analysis of these constructions seems most likely given their productivity, but diagnostics developed in this paper will suggest that lexical verbs in VV single event constructions must be in a relationship of adjunction rather than complementation to one another.

I consider these analyses to be on the right track, in that the relationship of -ip-marked material to V2 is likely one of adjunction when V2 is a lexical verb, but complementation when V2 is an auxiliary. However, I find it problematic to give an adverbial phrase a fixed position along the clausal spine, and I will briefly propose that -ip may be the head of one of two event phrases in section 8.3.

The greater issue is that Tohti and Abdurusul's analyses consider -ip to be the same kind of head in all cases, selecting a verbal constituent and adjoining to a VP in the case of multiple event constructions, or serving as the complement of an auxiliary phrase in auxiliary or even VV single event constructions. In this paper, I show through four diagnostic tests that multiple event constructions, VV single event constructions, and auxiliary constructions each have different underlying structures, with -ip selecting a different constituent and either adjoining to a different site or occupying a different clausal position in each construction.

3. Objectives. In this paper, I propose that -ip constructions in Uyghur can be divided according to their behavior under four structural tests. More specifically, a tripartite division emerges based on whether a construction shows uniformly monoclausal behavior (containing a single $\mathrm{TP}$ ), uniformly multiclausal behavior (involving high adjunction of a TP), or mixed behavior (involving low adjunction below the $v$ phase head). The results are summarized in table 1 .

I will use the results of these tests to make initial arguments that -ip may be a non-finite $\mathrm{T}$ head in multiple event constructions, a low event head in VV single event constructions, or a high or low event head in auxiliary constructions.

In the sections that follow, I will walk through the results of each test applied to each of the 


\begin{tabular}{cccc} 
& Multiple Event & VV Single Event & Auxiliary \\
\hline Single Passive Marking & - & + & + \\
\hline Single Negation & - & + & + \\
\hline NPI Subject Licensing & - & - & + \\
\hline NPI Adverbial Licensing & - & - & + \\
\hline Proposed Structure & TP adjunction & Low adjunction & Monoclausal \\
\hline
\end{tabular}

Table 1: Results of four structural diagnostics applied to three types of -ip constructions and structures to be proposed (' + ' indicates a given test yields a positive result)

three -ip constructions. Section 4 discusses the ability of passive morphology on V2 to render the entire construction passive, and section 5 compares whether negation on V2 negates the entire construction. The next two sections discuss the results of two NPI tests applied to -ip constructions: section 6 examines the ability of negation of V1 to license an NPI subject, and section 7 examines the ability of negation of V2 to license an NPI adverbial that appears before both verbs. In section 8 , I point to possible analyses of -ip that are developed in forthcoming work, and I conclude in section 9.

4. Single passive marking. I assume a clause to have one passive marker available, since the passive marker is the realization of a voice (or $v$ ) head with a single, fixed position in the clausal spine (Cinque 1999). Under this assumption, I predict that it should only be possible to passivize the higher of two verbs if both verbs are under the same passive voice head and/or appear in the same clausal spine.

Example (8) shows that a multiple event construction requires passive morphology on all transitive verbs to yield a passive reading, while (9) shows that a VV single event construction allows only one passive marker to appear (on V2) in order to yield a passive reading of two transitive verbs.

$$
\begin{array}{llllll}
\text { Her } & \text { yil-i } & \text { yéngi } & \text { söz-lar lughet-i } & \text { tüz-*(il)-ip } & \text { neshr } \\
\text { Every } & \text { year-3.POSS } & \text { new } & \text { word-PL dictionary-3.POSS } & \text { compile-PASS-IP publish } \\
\text { qil-*(in)-gan } & & & & \\
\text { do-*PASS-PERF } & & &
\end{array}
$$

"Every year a new dictionary has been compiled and published." (Dawut 2011: 96)

(9) Mital ur-(*il)-ip tüzli-wet-il-di-0

Metal hit-*PASS-IP flatten-RES-PASS-PST-3

"The metal was beaten flat." (A. Tash, p.c., 8/24/2016)

For auxiliary constructions as well, only one passive marker is allowed in passive sentences. Notice that there is some variation as to whether the passive marker attaches to V1 (as in (10)), or to the auxiliary itself (as in (11)), depending on the auxiliary used. One possible explanation of this variation will be mentioned in section 8.3.

$$
\begin{array}{lll}
\text { Telewizor buz-il-ip } & \text { qal- }(* \text { in)-di-0 } \\
\text { TV } & \text { break-PASS-IP } & \text { remain-*PASS-PST-3 } \\
\text { "A story was told to us." (L. Tohti, p.c., 11/30/2016) }
\end{array}
$$


Bir hikaye biz-ga sözle-(*in)-ip ber-il-di-0

One story 1PL-DAT speak-*PASS-IP give-PASS-pst-3

"The TV was broken." (Engesæth et al 2010: 237)

The patterns seen in this section suggest that there are two voice heads present in multiple event constructions, while only one voice head is present in VV single event and auxiliary constructions. There is also some variability as to which auxiliaries (along with -ip) are structurally higher versus lower than the passive voice head.

5. Single negation. Under the assumption that a negative head can negate any predication under its c-command, I predict that a negative marker attached to V2 should negate both verbs unless $\mathrm{V} 1$ is adjoined higher than sentential negation.

In all -ip constructions, it is possible to negate either verbal constituent. Negation of V1, however, only negates V1 and never negates V2 in any cases. When V1 is negated, a negative counterpart -may appears in place of -ip. ${ }^{3}$ (12), (13), and (14) show examples of multiple event, VV single event and auxiliary constructions with negation on V1, respectively. Note from the translations that in all cases V1 is understood as negated, but the sentence still has a positive reading because $\mathrm{V} 2$ is outside the scope of negation.

(12) Aldiri-may sözle-ing-e

Busy-NEG speak-IMP.2SG-EMPH

"Please don't speak in a hurry!" (Tohti 2012: 375)

Gep qil-may jim oltur-sa-ng-chu

Word do-NEG silent sit-COND-2SG-EMPH

"Please sit down without making a sound." (Tohti 2012: 372)

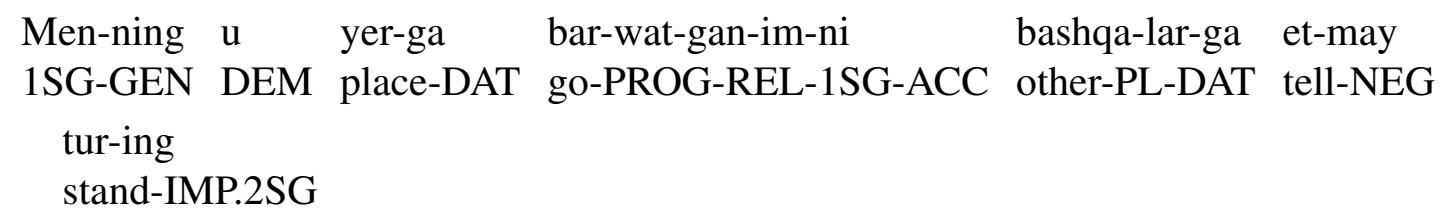

"Don't yet tell others that I have been going there (keep not lettting others know)." (5000 common words, Anki File)

Negating V1 thus is not an interesting diagnostic of clausal structure in -ip constructions, although it makes clear that V2 is structurally superior to V1. Negating V2, however, reveals differences in the scope of negation.

Negating V2 in multiple event constructions can negate the V2 constituent while still allowing $\mathrm{V} 1$ to have a positive reading, as shown in (15).

(15) Ular meydan-da putbol oyna-ip yataq-ga qayt-ma-di-0 3PL field-LOC soccer play-IP dorm-DAT return-NEG-PST-3

"They played soccer on the field, and/but didn't come back to the dorm." (Yüyán fängxiàng WeChat Group, 11/25/2016)

In VV single event constructions, on the other hand, negating V2 necessarily negates V1 to yield

\footnotetext{
${ }^{3}$-may is morphologically related to the finite verbal negation marker -ma. Abdurusul (2014) analyzes it as a phonological fusion of - $m a$ followed by -ip.
} 
an overall negative reading of the sentence, as seen in (16).

Tursun qayt-ip kel-ma-di-0

NAME return-IP come-NEG-PST-3

“Tursun didn't come back." (Engesæth 2000: 44)

Auxiliary constructions behave like VV single event constructions in that a negative marker attached to V2 also negates V1. (17), for example, means that the speaker did not write a novel.

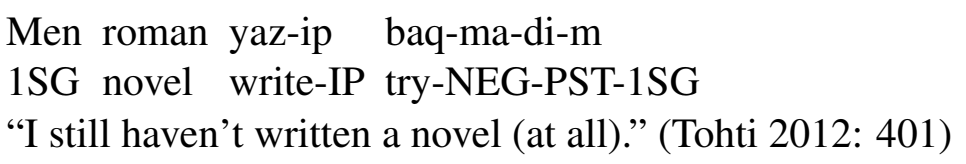

This section has shown that V1 is under the scope of negation of V2 in VV single event and auxiliary constructions, but not in multiple event constructions. This suggests that V1 may be adjoined to a higher position than sentential negation. V1P in single event and auxiliary constructions may either be a complement of V2 or adjoined lower than NegP.

6. NPI subject licensing. In the related (by hypothesis) Altaic language Korean, Choi (2003) shows that NPI subjects must occur in same clause as negation, and uses this diagnostic to distinguish a family of constructions quite similar to Uyghur -ip constructions. In Uyghur, it is also the case that NPI subjects must be generated in the same clause as negation to be licensed. (18) is ruled out because the negative suffix - $m a$ is in the embedded clause, while the NPI héchkim is the subject of the matrix clause.

$$
\begin{array}{lllll}
* \text { Héchkim bu kino-ni } & \text { kör-ma-y-men } & \text { de-di-0 } \\
\text { Nobody } & \text { DEM movie-ACC } & \text { watch-NEG-NPST-1SG } & \text { say-PST-3 }
\end{array}
$$

Intended: "Nobody said, 'I'm not going to watch this movie'." = "Nobody said they're not going to watch this movie." (M. Yakup, p.c., 6/19/2015)

I predict that if negation of either verb in an -ip construction can license an NPI subject, then both verbs must be located in the same clausal spine, with the subject base-merged lower than both positions of negation.

(19), (20) and (21) provide examples of V2 negation with an NPI subject in multiple event, VV single event and auxiliary constructions, respectively.

(19) Héchkim meydan-da putbol oyna-ip yataq-qa qayt-ma-di-0.

Nobody field-LOC football play-IP dorm-DAT return-NEG-PST-3

"Nobody played soccer on the field and returned to the dorm." (A. Tash, p.c., 8/24/2016)

(20) Héchkim mital-ni ur-ip tüzle-wet-ma-di-0.

Nobody metal-ACC hit-IP flatten-RES-NEG-PST-3

"Nobody hammered the metal flat." (A. Tash, p.c., 8/24/2016)

(21) Héchkim aghr-ip qal-ma-di-0.

Nobody get.sick-IP remain-NEG-PST-3

"Nobody got sick." (A. Tash, p.c., 8/24/2016)

The fact that an NPI subject is always licensed by negation of V2 tells us that V2 is always on the main clausal spine, and that subjects are generated under the c-command of the sentential negation marker -ma. -Ip constructions show variable behavior, however, in terms of whether 
the negative counterpart of -ip on V1 can license an NPI subject.

Sentences (22) and (23) show that negation of V1 does not license the NPI subject héchkim 'nobody' in multiple event and VV single event constructions, respectively.
* Héchkim tamaq ye-may öy-ga qayt-di-0
Nobody food eat-NEG.IP home-DAT return-PST-3
Intended: "Nobody ate food and returned home." (A. Turdimemet, p.c., 12/9/2016)
$\begin{array}{clll}* \text { Héchkim yataq-ga kir-may } & \text { kel-di-0 } \\ \text { Nobody dorm-DAT enter-NEG } & \text { come-PST-3 }\end{array}$
Intended: "They were pretty busy, so everybody came without going into the dorm." (A. Turdimemet, p.c., 12/9/2016)

Auxiliary constructions, however, allow a subject to be licensed by negation of V1, as shown in (24).

$$
\begin{aligned}
& \text { Waqt-i bol-ma-gan-liq-i üchün, héchkim bar-may qoy-di-0 } \\
& \text { Time-3.POSS be-NEG-PERF-NMLZ-3.POSS for nobody go-NEG.IP put-PST-3 } \\
& \text { "Because they didn't have time, nobody went home." (L. Tohti, p.c., 7/28/2016) }
\end{aligned}
$$

We have seen in this section that auxiliary constructions, but not multiple event or VV single event constructions, allow an NPI subject to be licensed by negation of V1. Presumably, acceptability of an NPI subject means that the subject is base-merged under the c-command of V1's negation, or at least in the same clause (Kim 2001). Inability to license the NPI subject indicates that V1 and its negation are adjoined to the matrix clause, rather than part of the main clausal spine.

7. NPI adverbial licensing. A final structural test involves the NPI adverbial anche. The adverbial anche must co-occur in the same clause as a negated verb, and the combination of anche plus verb $\mathrm{X}$ yields the meaning that the subject didn't perform the action denoted by X very much, or only performed the action to a minor extent (Tohti 2012).

Anche used in combination with a negated V1 is always acceptable. As (25) exemplifies, the reading in a multiple event construction is that the action of V1 wasn't carried out to a great extent, and then V2 was performed.

$\mathrm{U}$ anche köp ye-may-la öy-ga qayt-ip ket-di-0

3SG so.much much eat-NEG-EMPH house-DAT return-IP leave-PST-3

“(S)he didn't eat that much, and returned home." (C. Abdilim, p.c., 5/9/2017)

In single event constructions like (26), negating V1 with anche means that the action of V2 was carried out while barely performing the subevent of V1.

Batur mital-ni anche köp uru-may-la tüzle-wet-di-0

NAME metal-ACC so.much much hit-NEG-EMPH flatten-RES-PST-3

"Batur flattened the metal without hitting it so much." (C. Abdilim, p.c., 5/9/2017)

When V1 is negated and preceded by anche in an auxiliary construction, the reading is that the action of V1 is performed only to a minor extent, and the grammatical meaning of the auxiliary is not negated. Thus (27) means that the subject continues to write letters to the speaker only rarely. 
U men-ga anche köp xet yaz-may tur-wat-y-du 3SG 1SG-DAT so.much many letter write-NEG stand-PROG-NPST-3

"(S)he keeps not writing me that many letters." (C. Abdilim, p.c., 5/9/2017)

It is also possible for $\mathrm{V} 2$ to license anche when anche appears between the two verbal constituents in multiple (28) and VV single event constructions (29). Example (28) means that the subject came (to some event) and didn't eat that much, while (29) means that Batur hit the metal, but didn't make it that flat as a result.

U kech-raq kel-ip anche jiq ye-ma-di-0

3SG late-more come-IP so.much much eat-NEG-PST-3

“(S)he came late and didn't eat that much.” (C. Abdilim, p.c., 5/9/2017)

Batur mital-ni uru-ip anche tüzle-wet-ma-di-0

NAME metal-ACC hit-IP so.much flatten-RES-NEG-PST-3

"Batur didn't flatten the metal that much by hitting it." (C. Abdilim, p.c., 5/9/2017)

It is not possible, however, for anche to appear between $\mathrm{V} 1$ and $\mathrm{V} 2$ in an auxiliary construction.

$\begin{array}{lllll}* \mathrm{U} & \text { men-ga köp xet yaz-ip anche tur-may-wat-y-du } \\ \text { 3SG } & \text { 1SG-DAT many letter write-IP so.much stand-NEG-PROG-NPST-3 }\end{array}$

Intended: "(S)he doesn't keep writing me that many letters." (C. Abdilim, p.c., 5/9/2017)

That the NPI adverbial can be licensed by negation of the immediately adjacent verb (with the exception of V2 in auxiliary constructions) is to be expected if NPIs are licensed by being clausemates with a negative head. It would only be expected that negation of V2 could license anche despite the two constituents being separated by V1 if all the constituents were on the same clausal spine, however.

(31) and (32) show that negation of V2 cannot license anche when it appears before V1 in multiple event and VV single event constructions, respectively.

$\begin{array}{llll}* \mathrm{U} & \text { anche } & \text { (köp) ye-ip öy-ga } & \text { qayt-ma-di-0 } \\ \text { 3SG } & \text { so.much much eat-IP home-DAT } & \text { return-NEG-PST-3 }\end{array}$

Intended: “(S)he didn't really eat and go home.” (A. Turdimemet, p.c., 11/24/2016)

*U yataq-ga anche köp kir-ip kel-ma-y-du

3SG dorm-DAT so.much often enter-IP come-NEG-NPST-3

Intended: "(S)he doesn't come into the dorm that much.” (A. Turdimemet, p.c., 11/24/2016)

However, negation of V2 can license anche in an auxiliary construction, as indicated by the grammaticality of (33).

Ular men-ga anche köp xet yaz-ip tur-ma-y-du 3PL 1SG-DAT so.much many letter write-IP stand-NEG-NPST-3

"They don't write me letters that often." (A. Turdimemet,, p.c., 12/1/2016)

This section has shown that it is possible for V2 to license the NPI adverbial anche in pre-V1 position in auxiliary constructions, but not in multiple event or VV single event constructions. This constraint can be explained straightforwardly on the assumption that anche has a fixed position close to $v$ on the clausal spine. If $\mathrm{V} 1$ is in an adjunct in multiple event and VV single 
event constructions, then the only way for anche to appear before V1 is if it is part of the same adjunct, unable to be clausemates with negation over V2. The monoclausality of auxiliary constructions and fixed position of anche explains how negation of the higher V2 can license an NPI adverbial before lower V1, and anche cannot appear between V1 and V2.

8. Towards an analysis. The diagnostics discussed in this paper show that -ip is used in structurally distinct constructions, making a uniform analysis of -ip as an adverbializing head untenable. If we wish to analyze -ip as a functional head, then it cannot be occupying the same functional head in all cases. The diagnostics used here have suggested that a projection headed by -ip is adjoining to the main clause in some cases, and being selected as a complement by V2 within the same clause in others. Here I sketch possible analyses of -ip to be further developed in future work, based on the division of three structurally distinct -ip-constructions uncovered in this paper.

8.1 A DISTRIBUTED MORPHOLOGY ACCOUNT OF -ip. The variety of positions in which -ip may appear might lead one to argue that this morpheme does not correspond to any syntactic head in the clausal spine. This idea is proposed by Meral (2012), who observed that -ip's counterpart in Kazakh appears in free distribution with another morpheme, $-e$. The apparent lack of correspondence of what he calls converb markers to any aspectual meaning leads Meral to propose that -ip and $-e$ are "disassociated morphemes" in the sense of Embick and Halle (2005), inserted at PF for morphological wellformedness when a lexical verb appears adjacent to an auxiliary. 4

This idea avoids the complications of -ip's role in structurally distinct constructions, but begs the question of why such a morphological wellformedness requirement exists in Turkic languages. One might also take the existence of a negative counterpart to -ip as evidence that there is some syntactic head that it fills. The discussion below offers glimpses of potential analyses for -ip as a syntactic head in the three constructions under discussion.

8.2 -ip AS A T HEAD IN MULTIPLE EVENT CONSTRUCTIONS. Multiple event constructions showed multiclausal, adjunction-related behavior under the diagnostics applied. In these constructions, V1 and V2 may select separate objects marked with accusative case or even separate subjects, suggesting that a large amount of structure is attached to -ip. Section 4 already explained that V1 and V2 may each take a separate passive voice suffix, suggesting the constituent selected by -ip at least contains a Voice head. Additionally, as shown in (34), V1 may take the progressive -wat suffix, which is structurally higher than $v \mathrm{P}$.

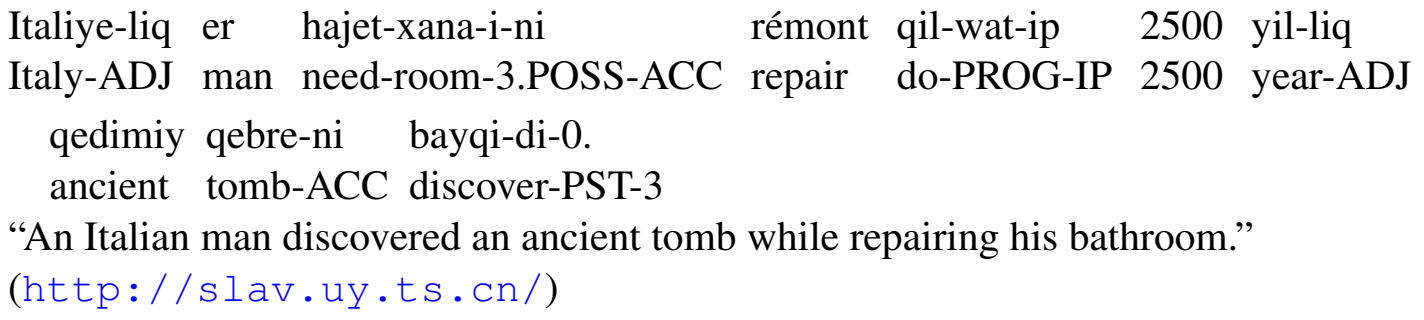

These facts, along with -ip's complementary distribution with finite marking (Bridges 2008), suggest that -ip may be a non-finite $\mathrm{T}$ head, selected by a separate $\mathrm{C}$ head when separate subjects are present. A simple derivation of (34) is sketched in (35).

\footnotetext{
${ }^{4}$ This suggestion echoes a similar idea mentioned by Bridges (2008).
} 


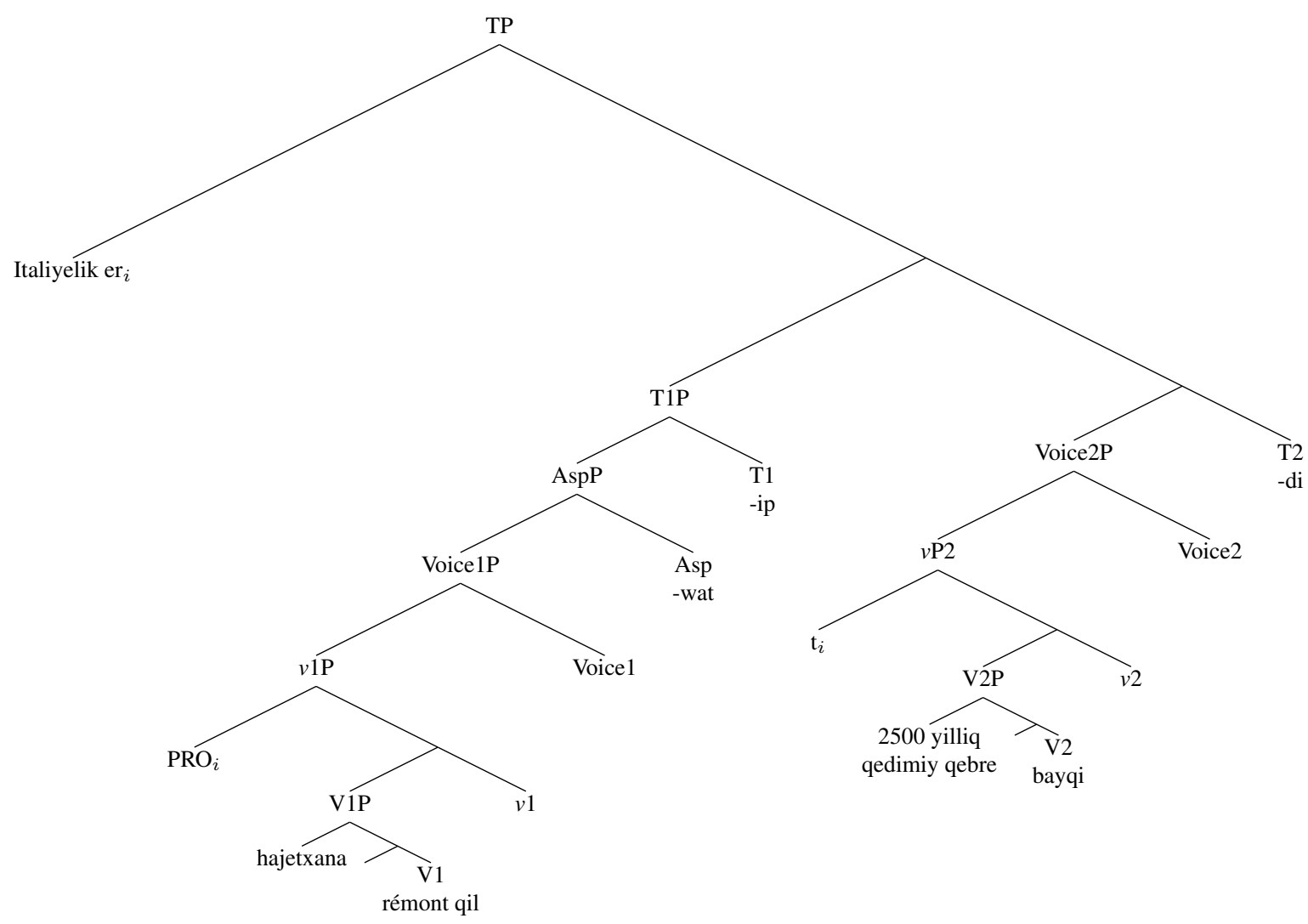

\section{3 -ip AS HIGH OR LOW EVENT HEADS IN AUXILIARY CONSTRUCTIONS. Auxiliary} constructions were found to be monoclausal with respect to all four tests. The uniformly monoclausal behavior of this construction can be explained if V1 is a lexical V head, while V2 is an auxiliary head somewhere higher in the clausal spine.

Recall from section 4, however, that auxiliary constructions were not uniform with the respect to the position of -ip and the auxiliary vis-a-vis the passive voice marker -il. Certain auxiliaries (along with -ip) always appear before the passive marker, while other auxiliaries always appear after the passive marker. Furthermore, as discussed in Sugar (unpublished), there appear to be generalizations to be made about the aspectual properties and argument structure of auxiliaries that appear lower vs. higher than passive voice in the clausal structure. Informally, auxiliaries that appear below voice are associated with durative, telic events performed by an agent, while auxiliaries that appear above voice are associated with achievements, iterated or incompleted events, and/or events lacking an agent.

Fukuda (2012) also uses the location of passive morphology to propose that Japanese aspectual verbs may either occupy an aspectual head internal to $v \mathrm{P}$ or a higher aspectual head just outside of $v \mathrm{P}$, in the spirit of Travis (1991). While there is information expressed by auxiliaries not related to aspect that makes calling them pure aspect heads undesirable (Bridges 2008, Sugar 2016), it is equally clear that auxiliaries are associated with high and low positions vis-a-vis voice. Auxiliaries that appear above voice are associated with either the beginning or iteration of events, I suggest that the -ip that appears above voice is an event head of the type proposed by Travis (2010). Those auxiliaries that appear below passive voice take external arguments, 
suggesting that they may be flavors of $v$. However, section 5 also showed that V1 can be negated in auxiliary constructions. Causative morphology may also appear below -ip and one of the alleged $v$ phase heads. For this to be possible, I suggest that lexical verbs are complement to a defective $v$, embedded under the $v$ (in the sense of Miyagawa 1998) that serves as a phase head. -Ip then fills a lower event head between $v$ layers.

The clausal spine described here is shown in (36).

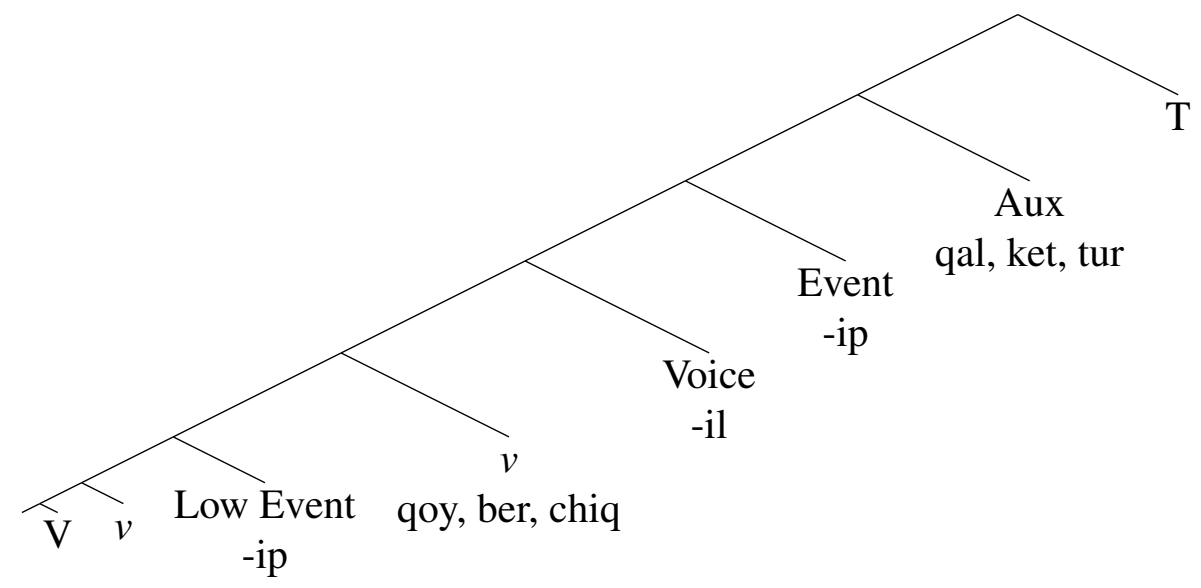

8.4 -ip AS AN EVENT HEAD IN VV SINGLE EVENT CONSTRUCTIONS. VV single event constructions showed some adjunction behavior, but they share objects and are under the scope of the same passive and negation heads, suggesting that adjunction happens lower than Voice. In the same spirit as the analysis just described of auxiliary constructions, I suggest that the adjoined constituent may be an -ip-headed event phrase whose complement is a defective $v \mathrm{P}$. The defective $v$ does not introduce its own external argument, and object sharing happens by control. (38) shows a possible derivation of (37).

Tursun qayt-ip kel-di-0

NAME return-IP come-PST-3

"Tursun came back." (A. Turdimemet, p.c., 12/9/2016) 


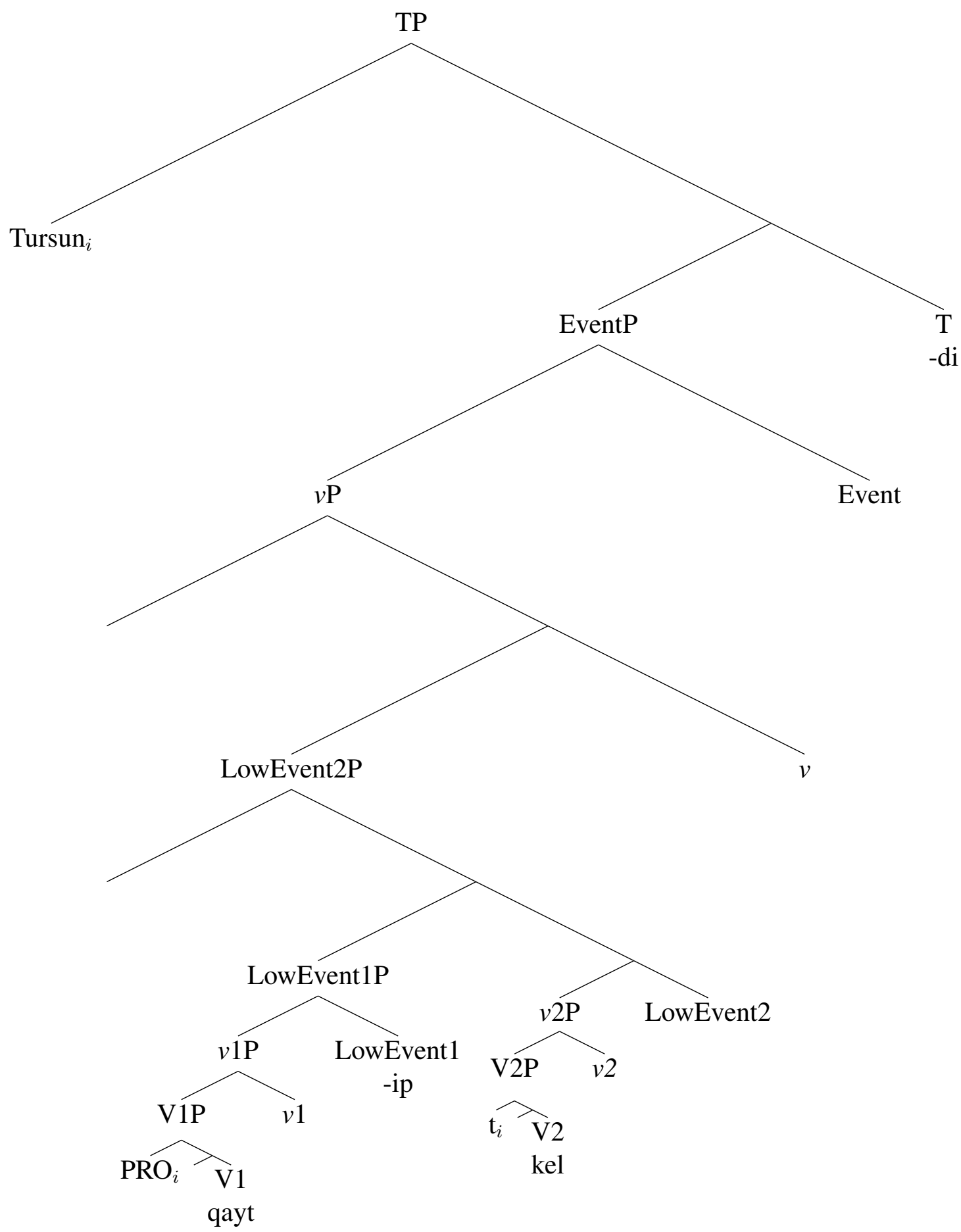

This and the other analyses presented in this section are intended as possible directions of future study in developing a comprehensive analysis of -ip constructions in Uyghur and the phenomenon of multi-verb constructions cross-linguistically.

9. Conclusion. This paper has provided four structural diagnostics to adjudicate between structures involving high or low adjunction and a monoclausal spine. These diagnostics show that the -ip suffix in Uyghur must not be the same head in the same structure when it appears in semantically different constructions (multiple event constructions, single event constructions 
involving two lexical verbs, and auxiliary constructions). Multiple event constructions behaved differently from auxiliary constructions under all four tests. VV single event constructions patterned with multiple event constructions in disallowing licensing of an NPI subject by negation of V1 or licensing of an NPI adverbila by negation of V2, but patterned with auxiliary constructions in allowing only one passive marker and having single negation. I have proposed to capture the similarities between multiple event constructions and VV single event constructions by arguing that these constructions involve adjunction while auxiliary constructions involve complementation, and the similarities between VV single event constructions and auxiliary constructions by arguing that in these constructions -ip is an event head, while in multiple event constructions it is a T head. These proposed structures will be further explored in future work.

\section{References}

Abdurusul, Muzappar. 2014. Weiwueryu de jingcihua duanyu. Beijing: Minzu Press. Bridges, Michelle. 2008. Auxiliary Verbs in Uyghur. MA Thesis: University of Kansas.

Choi, Seongsook. 2003. Serial verbs and the empty category. In Dorothee Beermann and Lars Hellan (eds.), Proceedings of the workshop on Multi-verb constructions. Trondheim: Norweigian University of Science and Technology.

Cinque, Guglielmo. 1999. Adverbs and Functional Heads. New York: Oxford University Press. Dawut, Ömer. 2011. Uyghur tili medeniyiti tetqiqati. Urumchi: Xinjiang University Press. Embick, David and Morris Halle. 2005. On the Status of Stems in Morphological Theory. In Twan Geerts, Ivo Ginneken and Haike Jacobs (eds.), Proceedings of Going Romance 2003. Amsterdam and Philadelphia: John Benjamins. 37-62.

Engesæth, Tarjei, Mahire Yakup and Arienne Dwyer. 2010. Greetings from the Teklimkana: a Handbook of Modern Uyghur Volume 1. Lawrence: University of Kansas Scholarworks.

Fukuda, Shin. 2012. Aspectual verbs as functional heads: Evidence from Japanese aspectual verbs. Natural Language \& Linguistic Theory 30(4): 965-1026.

Ibrahim, Ablahat. 1995. Meaning and Usage of Compound Verbs in Modern Uighur and Uzbek. PhD Diss., University of Washington.

Kim, Kyoung-Kook. 2001. Korean negation and the licensing condition on negative polarity items. PhD Diss., University of Hawai'i.

Meral, Hasan Mesut. 2012. Kazakh complex verb structures: a Distributed Morphology analysis. Turkic languages 16(2): 239-256.

Miyagawa, Shigeru. 1998.(S)ase as an Elsewhere causative and the syntactic nature of words. Journal of Japanese Linguistics 16: 67?110.

Sugar, Alexander. 2016. Classifying Uyghur Light Verbs by Situation Aspect Type for Use in a Precision Grammar. Generals paper, University of Washington.

- . -ip as High and Low Event Heads in Uyghur. Unpublished manuscript, University of Washington.

Tohti, Litip. 2012. Xiandai weiwueryu cankao yufa. Beijing: China Social Sciences Press. Tömür, Hamit. 2003. Modern Uyghur Grammar: Morphology. Trans. Anne Lee. Istanbul, Turkey: Yildiz.

Travis, Lisa M. 1991. Derived object, inner aspect, and structure of VP. Paper presented at NELS 22. October, 1991.

- 2010. Inner Aspect. New York, NY: Springer. 